Ann. Biol. anim., Bioch., Biophys., I965, 5 (4), 469-48г.

\title{
DÉTERMINATION DU BESOIN EN ACIDES AMINÉS SOUFRÉS CHEZ LE RAT BLANC EN CROISSANCE
}

\author{
A. RÉRAT et Y. HENRY \\ avec la collaboration technique de Françoise Houlier, G. Rouzet et M. Lecourtier \\ Station de Recherches sur l'Élevage des Porcs, \\ Centre national de Recherches zootechniques, Jouy-en-Josas (Soine-et-Oise)
}

SOMMAIRE

Le besoin en acides aminés soufrés du Rat blanc en croissance a été déterminé au cours de deux expériences, basées sur la supplémentation par la DL-méthionine de régimes renfermant des proportions variables d'une farine de hareng de Norvège. L'addition de méthionine aux protéines de poisson entraîne une amélioration significative de la vitesse de croissance et de la rétention azotée à des taux de matières azotées inférieurs ou égaux à I 2 p. Ioo. La quantité d'acides aminés soufrés (méthionine + cystine) nécessaire pour l'équilibre du régime décroît, relativement aux protéines, lorsque le taux de ces dernières augmente : respectivement 5 et $4-4,5$ p. Ioo pour 6 et I 2 p. I0O de protéines. Le besoin correspondant à la vitesse de croissance maximum $(5,4$ à $5,7 \mathrm{~g} / \mathrm{j}$ après 28 jours), représente entre 0,49 et $0,54 \mathrm{p}$. 100 d'un régime renfermant $4150 \mathrm{Kcal}$ digestibles $/ \mathrm{kg}$, soit en moyenne 70 à $75 \mathrm{mg}$ par jour entre les poids de $5 \circ \mathrm{g}$ et $300 \mathrm{~g}$.

\section{INTRODUCTION}

Le besoin en amino acides indispensables chez l'animal en croissance a fait l'objet d'un grand nombre de travaux basés sur la supplémentation d'un régime par le facteur limitant à des niveaux variables, les critères retenus étant généralement la vitesse de croissance et la rétention azotée. Parmi les méthodes mises en œuvre pour déterminer le niveau de ce besoin, certaines utilisent des régimes synthétiques renfermant un mélange d'acides aminés libres (RosE, I938; MERTz et al., I952). D'autres font appel à des régimes semi-synthétiques contenant une protéine naturelle déséquilibrée en la plupart des acides aminés indispensables (BRESSANI et MERTZ, I958) ou une protéine de bonne qualité à faible taux (RAO et al., I959), le complément en amino acides étant apporté sous forme libre afin de rétablir l'équilibre par rapport aux besoins ou vis-à-vis d'une protéine de référence. 
En réalité, toutes ces méthodes, dans lesquelles la totalité ou la majeure partie des acides aminés est introduite à l'état libre, sont relativement éloignées des conditions habituelles d'alimentation. C'est pourquoi nous avons été amenés à mettre au point une nouvelle méthode (RÉRAT et HENRY, I964), basée sur l'utilisation d'une protéine naturelle équilibrée en la plupart des acides aminés indispensables, mais présentant un déficit partiel de l'acide aminé dont on veut préciser le besoin. Appliquant cette technique, nous avons ainsi entrepris une expérimentation dans le but de déterminer le besoin en acides aminés soufrés (méthionine + cystine) chez le Rat en croissance, grâce à la supplémentation de la farine de poisson par la DL-méthionine de synthèse.

\section{TECHNIQUES EXPÉRIMENTAIES ET MATÉRIEL UTILISÉ}

Deux expériences successives sont réalisées avec des rats mâles de souche Wistar CF, pris au sevrage et élevés en cages individuelles. Elles ont pour objet de faire varier le taux de DL-méthionine supplémentaire dans des régimes semi-synthétiques renfermant des proportions variables d'une farine de hareng de Norvège. Cette dernière contient $90 \mathrm{p}$. Ioo de matière sèche et $72 \mathrm{p}$. 100 de matières azotées totales $(N \times 6,25)$. Sa composition en amino acides indispensables, rapportée à $\mathbf{I} 6 \mathrm{~g}$ d'azote ou à leur somme, selon Pion et al., (1963), est donnée dans le tableau i en comparaison

\section{TABLEAU I}

Composition de la farine de poisson en amino acides indispensables et comparaison avec les besoins du Rat

\begin{tabular}{|c|c|c|c|c|}
\hline & \multicolumn{2}{|c|}{ Farine de poisson } & \multirow{2}{*}{$\begin{array}{l}\text { Besoin du Rat } \\
\% \sum \mathrm{AAI}^{\left({ }^{3}\right)}\end{array}$} & \multirow{2}{*}{$\begin{array}{l}\text { Déficit } \% \\
\text { par rapjort } \\
\text { au besoin }\end{array}$} \\
\hline & $\begin{array}{l}\text { Quantités } \\
\text { en } g / 16 g \mathrm{~N}(1)\end{array}$ & $\% \quad \sum$ AAI & & \\
\hline Arginine $\ldots \ldots \ldots \ldots \ldots \ldots \ldots$ & $7,2(3,1)$ & 6,2 & 1,1 & - \\
\hline 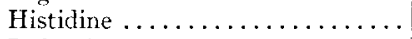 & 2,3 & 1,8 & 4,1 & $\longrightarrow$ \\
\hline Isoleucine. ................ & 5,1 & 10,6 & 11,4 & 7 \\
\hline Leucine ................ & $8,0(7,6)$ & 16,0 & 14,5 & - \\
\hline Lysine $\ldots \ldots \ldots \ldots \ldots \ldots$ & 7,7 & 16,0 & 18,7 & 14 \\
\hline 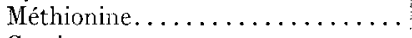 & 2,6 & & & \\
\hline Cystine...$\ldots \ldots \ldots \ldots \ldots \ldots$ & 0,9 & & & \\
\hline Méthionine + cystine ......... & 3,5 & 7,3 & 10,1 & 30 \\
\hline Phénylalanine ............. & 4,0 & & & \\
\hline Tyrosine $\ldots \ldots \ldots \ldots \ldots \ldots \ldots$ & 3,9 & & & \\
\hline Phénylalanine + tyrosine $\ldots .$. & 7,9 & $16,,^{\prime}$ & 15,9 & \\
\hline Thréonine $\ldots \ldots \ldots \ldots \ldots \ldots$ & 4,7 & 9,8 & 10,1 & \\
\hline Valine..$\ldots \ldots \ldots \ldots \ldots \ldots$ & 6,2 & 12,9 & 11,4 & \\
\hline$\sum A A I\left({ }^{2}\right)$. & 18,1 & & & \\
\hline
\end{tabular}

(1) Pion et al. (1963).

(2) $\Sigma$ AAI $=$ somme des acides aminés indispensables, selon FAUCONNEAU (196't) : le tryptophane, non dosé, a été exclus du calcul ; la leucine a été limitée à 150 p. 100 de l'isoleucine et l'arginine à $6,2 \mathrm{p} .100$ de la somme des acides aminés indispensables (chiffres entre parenthìses).

$\left({ }^{3}\right)$ D'après les estimations de Rao, Mffta et Jonnson (1959). 
avec les besoins définis par Rao, MEtTa et JonhSon (r959) exprimés en pourcentage de leur somme. L'examen de ce tableau fait apparaître un déficit primaire en acides aminés soufrés et un déficit secondaire en lysine. En dehors du fait que les acides aminés soufrés constituent le facteur limitant primaire de la farine de poisson, cette dernière a été choisie en raison de sa valeur biologique élevée et de l'excellente disponibilité de ses acides aminés (Geiger et Borgstrom, I962); en outre, elle présente l'avantage d'être peu sensible aux traitements thermiques qu'elle a subis au cours de sa préparation (RERAT et LOUGNON, 1963).

La première expérience $(\mathrm{A})$, d'une durée de 56 jours, comprend ${ }_{3} 3$ lots de 9 rats, dont ro sont répartis suivant un schéma factoriel $2 \times 5$ comportant :

- Deux taux de protéines : 8 et i 2 p. Ioo;

- Cinq taux de DL-méthionine supplémentaire exprimés en pourcentage des protéines : $0-0,2-0,4-0,6$ et 0,8 . Dans les 3 autres régimes, on introduit respectivement 10 , I4 et I6 p. Ioo de matières azotées, afin de suivre l'évolution de la croissance en fonction du taux protidique.

La deuxième expérience (B), d'une durée de 28 jours, porte sur 14 lots de 6 animaux dont 12 sont distribués suivant un dispositif factoriel $3 \times 4$ comprenant :

- Trois taux de matières azotées : 6, 9 et I 2 p. гоo ;

-- Quatre taux de DL-méthionine supplémentaire exprimés en pourcentage des protéines : $0-0,5-1,0$ et $\mathrm{I}, 5$.

Deux taux de matières azotées supplémentaires ( 15 et I 8 p. Ioo) sont utilisés afin de préciser, comme dans l'expérience précédente, le taux correspondant à la vitesse de croissance maximum.

Ie schéma expérimental est résumé dans le tableau 2. La méthionine de synthèse est administrée sous la forme racémique DL. Pour que le taux d'azote aminé libre soit identique dans tous les régimes, on introduit un complément variable d'un acide aminé non indispensable (glycocolle).

\section{TABLEAU 2}

Schéma expérimental el teneur des régimes en acides aminés soufrés (en pourcentage de la ration)

Expérience A

\begin{tabular}{|c|c|c|c|c|c|}
\hline $\begin{array}{l}\text { DL-méthionine supplémentaire \% protéines. } \\
\text { Acides aminés soufrés totaux } \% \text { protéines.. }\end{array}$ & $\begin{array}{l}0 \\
3,5\end{array}$ & $\begin{array}{l}0,2 \\
3,7\end{array}$ & $\begin{array}{l}0, \mathbf{k}^{\prime} \\
3,9\end{array}$ & $\begin{array}{l}0,6 \\
4,1\end{array}$ & $\begin{array}{l}0,8 \\
4,3\end{array}$ \\
\hline & \multicolumn{5}{|c|}{ Acides aminés soufrés totaux \% régime } \\
\hline 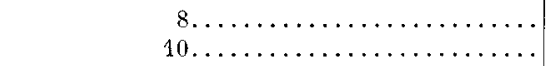 & $\begin{array}{l}0,28 \\
0,35\end{array}$ & 0,30 & 0,31 & 0,33 & 0,34 \\
\hline $\begin{array}{rl}\text { Protéines } \% & 12 \ldots \ldots \ldots \ldots \ldots \ldots \ldots \ldots \ldots \\
& 14 \ldots \ldots \ldots \ldots \ldots \ldots \ldots \ldots \\
& 16 \ldots \ldots \ldots \ldots \ldots \ldots \ldots \ldots\end{array}$ & $\begin{array}{l}0,42 \\
0,49 \\
0,56\end{array}$ & 0,44 & 0,47 & 0,49 & 0,52 \\
\hline
\end{tabular}

Expérience B

DL-méthionine supplémentaire \% protéines . Acides aminés soufrés \% protéines ........

Protéines \%

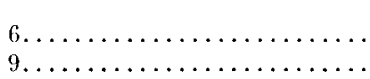

0 3,5 Acides aminés soufrés totaux \% régime

\begin{tabular}{l|l|l|l|}
0,21 & 0,24 & 0,27 & 0,30 \\
0,31 & 0,36 & 0,40 & 0,45 \\
0,42 & 0,48 & 0,54 & 0,60 \\
0,52 & & & \\
0,63 & & &
\end{tabular}

In dehors de la farine de poisson, tous les régimes renferment 8 p. Ioo d'huile d'arachide additionnée de vitamines A et D, 2 p. Ioo de cellulose de bois, 3 p. Ioo de mélange minéral et i p. roo de mélange vitaminique, le complément étant apporté sous forme d'amidon de maïs. La valeur énergétique correspondante est de l'ordre de 4 I 50 Kilocalories digestibles par kg, d'après des résultats de digestibilité enregistrés par ailleurs (HENRY et RÉRAT, données non publiées). 
Les critères étudiés sont la vitesse de croissance, la rétention azotée et la composition corporelle. La rétention azotée est déterminée à partir des résultats d'analyse des carcasses, suivant une méthode exposée précédemment (HENRY et RÉRAT, I963). A ce sujet, il faut rappeler les définitions suivantes :

- Indice de consommation :

Matière sèche ingérée $(\mathrm{g}) / \mathrm{gain}$ de poids $(\mathrm{g})$.

- Coefficient d'efficacité protidique (C. E. P.) :

Gain de poids (g)/protéines ingérées $(\mathrm{g})$.

-. Coefficient d'utilisation pratique de l'azote (C. U. P.) :

$\mathrm{N}$ retenu dans les tissus $(\mathrm{g}) \times$ Ioo/N ingéré $(\mathrm{g})$, selon Terroine et Valda (I933).

\section{RÉSULTATS}

\section{Expérience $A$}

L'expérience $B$ ayant duré 28 jours, les rést1ltats de 1'expérience $A$ sont donnés après 28 jours (tabl. 3 ) et 56 jours (tabl. 4 ).

\section{TABLEAU 3}

Expérience A: Résultats généraux de croissance et de consommation après 28 jours - Poids moyen initial : $70,7 \mathrm{~g}$

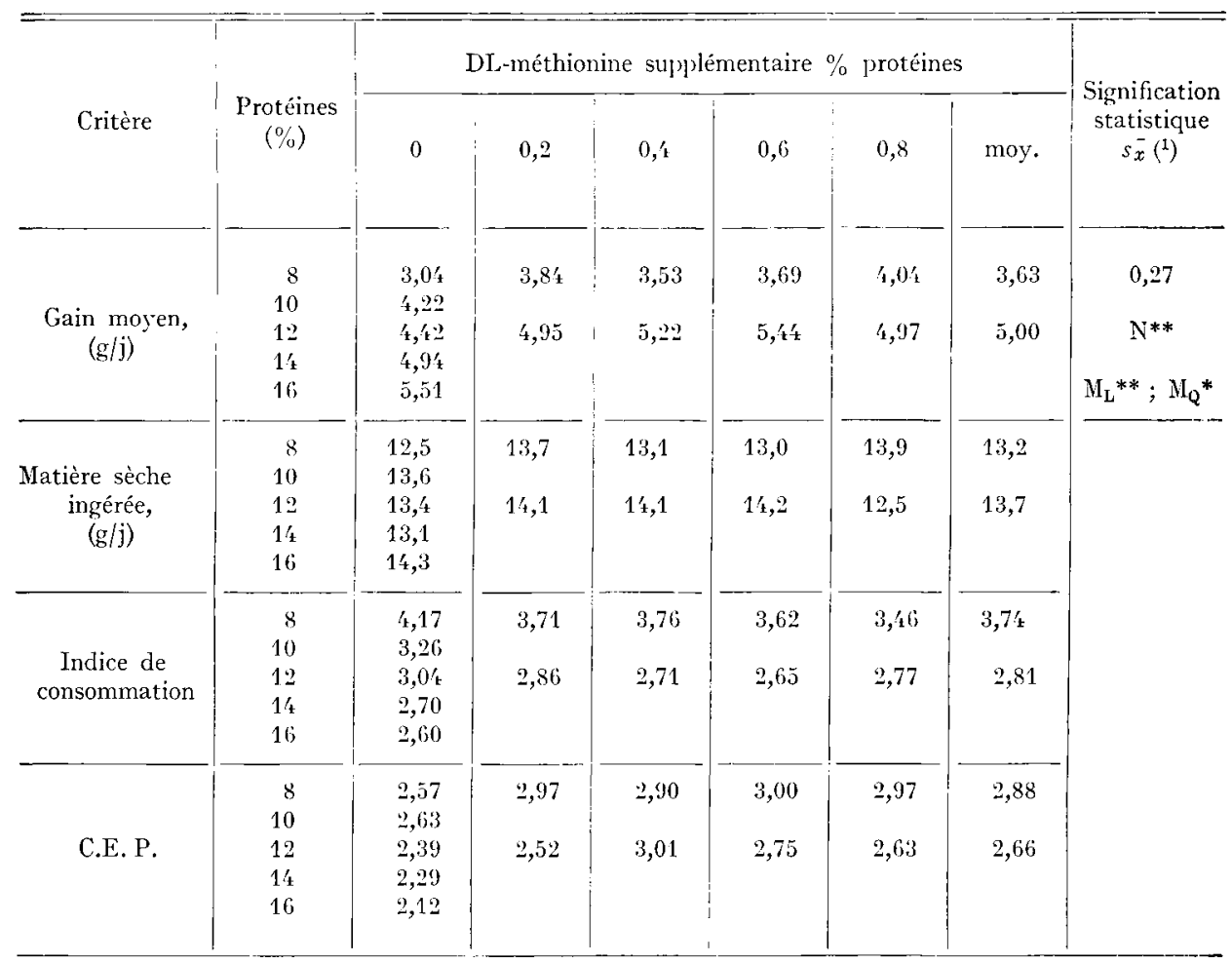

(1) $s_{x}$ : écart-type de la moyenne. $\mathrm{N}:$ taux azoté; $\mathbb{M}:$ taux de méthionine supplémentaire $\left(\mathrm{M}_{\mathrm{L}}:\right.$ terme linéaire; $\mathrm{M}_{\mathrm{Q}}$ : terme quadratique).

* : seuil de signification 0,$05 ; * *$ : seuil 0,01 .

Les calculs statistiques se rapportent uniquement au schéma factoriel. 
$\mathbf{I}^{\mathrm{O}}$ Résultats après 28 jours.

L'élévation du taux de matières azotées dans le régime entre 8 et I6 p. Ioo se traduit par une amélioration progressive de la vitesse de croissance qui passe de 3 à $5,5 \mathrm{~g}$ par jour. En même temps, la quantité de matière sèche ingérée quotidiennement augmente et l'indice de consommation diminue, le coefficient d'efficacité protidique (C. E. P.) atteint une valeur maximum pour un taux de matières azotées compris entre 8 et Io p. Ioo, puis diminue légèrement à mesure que le taux protidique augmente.

TABLEAU 4

Expérience A : Résultats généraux de croissance et de consommation après 56 jours

\begin{tabular}{|c|c|c|c|c|c|c|c|c|}
\hline \multirow{2}{*}{ Critire } & \multirow{2}{*}{$\begin{array}{c}\text { Protéines } \\
(\%)\end{array}$} & \multicolumn{6}{|c|}{ DL-méthionine supplémentaire \% protéines } & \multirow{2}{*}{$\begin{array}{c}\text { Signification } \\
\text { statistique } \\
\overline{s \bar{x}}\left({ }^{(1)}\right.\end{array}$} \\
\hline & & 0 & 0,2 & 0,4 & 0,6 & 0,8 & moy. & \\
\hline $\begin{array}{l}\text { Gain moyen } \\
(\mathrm{g} / \mathrm{j})\end{array}$ & $\begin{array}{r}8 \\
10 \\
12 \\
14 \\
16\end{array}$ & $\begin{array}{l}2,80 \\
3,67 \\
3,75 \\
4,1^{\prime} \\
4,71\end{array}$ & $\begin{array}{l}3,21 \\
4,30\end{array}$ & $\begin{array}{l}3,09 \\
4,61\end{array}$ & 4,62 & 4,53 & $\begin{array}{l}3,19 \\
4,36\end{array}$ & $\begin{array}{c}0,26 \\
\mathrm{~N}^{* *} \\
\mathrm{M}_{\mathrm{L}}^{* *}\end{array}$ \\
\hline M. S. ingérée & $\begin{array}{r}8 \\
10 \\
12 \\
14 \\
16\end{array}$ & $\begin{array}{l}13,8 \\
11,9 \\
14,4 \\
114,8 \\
16, y^{\prime},\end{array}$ & $\begin{array}{l}14,5 \\
15,5\end{array}$ & $\begin{array}{l}14,2 \\
15,8\end{array}$ & $\begin{array}{l}14,4 \\
15,7\end{array}$ & $\begin{array}{l}14,7 \\
15,5\end{array}$ & $\begin{array}{l}14,3 \\
15,4\end{array}$ & \\
\hline $\begin{array}{l}\text { Indice de } \\
\text { consommation }\end{array}$ & $\begin{array}{r}8 \\
10 \\
12 \\
1 / 4 \\
16\end{array}$ & $\begin{array}{l}4,96 \\
4,09 \\
3,86 \\
3,60 \\
3,51\end{array}$ & $\begin{array}{l}4,75 \\
3,71\end{array}$ & $\begin{array}{l}4,70 \\
3, y_{4} 4\end{array}$ & 3,50 & $\begin{array}{l}4,28 \\
3,45\end{array}$ & $\begin{array}{l}4,64 \\
3,59\end{array}$ & \\
\hline C. U. P. azoté & $\begin{array}{r}8 \\
10 \\
12 \\
114 \\
16\end{array}$ & $\begin{array}{l}39,2 \\
40,4 \\
36,3 \\
32,9 \\
30,3\end{array}$ & $\begin{array}{l}42,9 \\
36,3\end{array}$ & $\begin{array}{l}41,0 \\
40,3\end{array}$ & $\begin{array}{l}46,3 \\
39,8\end{array}$ & $\begin{array}{l}45,2 \\
40,4\end{array}$ & $\begin{array}{l}42,9 \\
38,6\end{array}$ & $\begin{array}{c}1,33 \\
\mathrm{~N}^{* *} \\
\mathrm{M}_{\mathrm{L}}{ }^{* *}\end{array}$ \\
\hline
\end{tabular}
linéaire).

(1) $s_{x}$ : écart-type de la moyenne. $\mathrm{N}$ : taux azoté ; $\mathrm{M}$ : taux de méthionine supplémentaire ( $\mathrm{M}_{\mathrm{L}}$ : terme

** : seuil 0,01 .

Les calculs statistiques se rapportent uniquement au schéma factoriel.

L'addition de DL-méthionine améliore l'ensemble des critères aux deux taux azotés considérés. La vitesse de croissance, au taux de 8 p. Ioo de protéines, répond favorablement à chaque addition d'extra-méthionine, tandis qu'au taux de I 2 p. Ioo elle présente une évolution d'allure parabolique avec un maximum pour o,6 p. Ioo de méthionine supplémentaire. Cependant, l'analyse statistique des résultats ne fait ressortir aucune interaction significative entre le taux de protéines et le niveau 
de supplémentation en méthionine. Le gain de poids maximum ainsi réalisé $(5,4 \mathrm{~g} / \mathrm{j})$ est sensiblement le même que celui obtenu avec le régime à $\mathrm{r} 6 \mathrm{p}$. Ioo de matières azotées, bien que la teneur en acides aminés soufrés obtenue grâce à la supplémentation soit plus faible (respectivement 0,49 et $0,56 \mathrm{p}$. Ioo du régime). Cette amélioration de la croissance s'accompagne d'une meilleure utilisation de la nourriture : 1'indice de consommation diminue et le $\mathrm{C}$. E. P. présente un maximum pour un taux compris entre 0,4 et $0,6 \mathrm{p}$. Ioo de DL-méthionine par rapport aux protéines.

$2^{\circ}$ Résultats après 56 jours.

L'évolution des critères est sensiblement la même que celle qui a été décrite après 28 jours. Remarquons cependant que la vitesse de croissance maximum $(4,6 \mathrm{~g} / \mathrm{j})$, plus faible que pendant les 4 premières semaines, est obtenue avec un taux d'extra-méthionine inférieur $(0,4$ p. Ioo). De même que le C. E. P., le coefficient d'utilisation pratique de 1'azote (C. U. P.) présente une évolution parabolique avec un maximum à 0,6 p. Ioo de méthionine supplémentaire pour 8 p. Ioo de protéines et à $0,4 \mathrm{p}$. Ioo pour I $2 \mathrm{p}$. Ioo de protéines, correspondant aux doses respective; de 0,33 et 0,47 p. roo d'acides aminés soufrés dans la ration.

Les modifications du taux azoté ou du taux de méthionine supplémentaire n'exercent aucun effet appréciable sur les pourcentages de protéines et de lipides dans les tissus (tabl. 5). Signalons toutefois un léger accroissement du pourcentage de protéines tissulaires entre 8 et Io p. Ioo de protéines dans le régime.

TABILAU 5

Expérience A : Résultats de composition corporelle

\begin{tabular}{|c|c|c|c|c|c|c|c|}
\hline \multirow{2}{*}{ Critère } & \multirow{2}{*}{$\begin{array}{c}\text { Protéines } \\
(\%)\end{array}$} & \multicolumn{6}{|c|}{ DL-méthionine supplémentaire $\%$ protéines } \\
\hline & & 0 & 0,2 & $0,,^{\prime}$ & 0,6 & 0,8 & moy. \\
\hline $\begin{array}{c}\text { Protéines } \\
(\%)\end{array}$ & $\begin{array}{r}8 \\
10 \\
12 \\
114 \\
16\end{array}$ & $\begin{array}{l}18,1 \\
19,0 \\
18,9 \\
19,0 \\
18,9\end{array}$ & 18,3 & 17,8 & 18,6 & 18,1 & $\begin{array}{l}17,8 \\
18,7\end{array}$ \\
\hline $\begin{array}{c}\text { Lipides } \\
(\%)\end{array}$ & $\begin{array}{r}8 \\
10 \\
12 \\
114 \\
18\end{array}$ & $\begin{array}{l}16,8 \\
16,4 \\
18,1 \\
17,5 \\
18,2\end{array}$ & 21,4 & 18,6 & 16,7 & 18,3 & $\begin{array}{l}18,3 \\
18,9\end{array}$ \\
\hline
\end{tabular}

Expérience $B$

Les résultats généraux de croissance et de consommation sont rapportés dans le tableau 6 . Il convient d'abord de remarquer que les performances des animaux sont sensiblement supérieures à celles enregistrées dans l'expérience précédente ; il s'agit de rats de la même souche, mais provenant d'un élevage différent. 
TABLEAU 6

Expérience B : Résultals généraux de croissance et de consommation. Poids moyen initial : $58,6 \mathrm{~g}$ - Durée : 28 jours

\begin{tabular}{|c|c|c|c|c|c|c|c|}
\hline & \multirow{2}{*}{$\begin{array}{c}\text { Protéines } \\
(\%)\end{array}$} & \multicolumn{5}{|c|}{ DL-Méthionine supplémentaire \% protéines } & \multirow{2}{*}{$\begin{array}{c}\text { Signification } \\
\text { statistique } \\
s=(1)\end{array}$} \\
\hline & & 0 & 0,5 & 1 & 1,5 & moyenne & \\
\hline $\begin{array}{c}\text { Gain moyen, } \\
(\mathrm{g} / \mathrm{j})\end{array}$ & $\begin{array}{c}6 \\
9 \\
12 \\
\text { Moy. } \\
15 \\
18\end{array}$ & $\begin{array}{l}2,23 \\
3,35 \\
4,89 \\
3,49 \\
5,58 \\
5,48\end{array}$ & $\begin{array}{l}2,33 \\
4,34 \\
4,71 \\
3,79\end{array}$ & $\begin{array}{l}2,60 \\
4,44 \\
5,73 \\
4,26\end{array}$ & $\begin{array}{l}3,07 \\
4,61 \\
5,56 \\
4,41\end{array}$ & $\begin{array}{l}2,56 \\
4,18 \\
5,22\end{array}$ & $\mathrm{~N}_{\mathrm{L}}{ }_{\mathrm{M}_{\mathrm{L}}}^{0,28} ; \mathrm{N}_{\mathrm{Q}}^{*}$ \\
\hline $\begin{array}{l}\text { M.S. ingérée, } \\
(\mathrm{g} / \mathrm{j})\end{array}$ & $\begin{array}{c}6 \\
9 \\
12 \\
\text { Moy. } \\
15 \\
18\end{array}$ & $\begin{array}{l}10,8 \\
12,0 \\
12,7 \\
11,8 \\
13,6 \\
13,0\end{array}$ & $\begin{array}{l}10,6 \\
12,9 \\
12,4 \\
12,0\end{array}$ & $\begin{array}{l}10,4 \\
12,5 \\
13,9 \\
12,3\end{array}$ & $\begin{array}{l}11,5 \\
12,7 \\
13,2 \\
12,5\end{array}$ & $\begin{array}{l}10,8 \\
12,5 \\
13,0\end{array}$ & $\begin{array}{c}0,62 \\
\mathrm{~N}_{\mathrm{L}}{ }^{* *}\end{array}$ \\
\hline $\begin{array}{c}\text { Indice de } \\
\text { consommation }\end{array}$ & $\begin{array}{c}6 \\
9 \\
12 \\
M o y . \\
15 \\
18\end{array}$ & $\begin{array}{l}5,11 \\
3,61 \\
2,59 \\
3,77 \\
2,45 \\
2,38\end{array}$ & $\begin{array}{l}4,72 \\
3,00 \\
2,66 \\
2,46\end{array}$ & $\begin{array}{l}4,04 \\
3,01 \\
2,43 \\
3,16\end{array}$ & $\begin{array}{l}4,00 \\
2,77 \\
2,39 \\
3,95\end{array}$ & $\begin{array}{l}4,47 \\
3,10 \\
2,52\end{array}$ & $\begin{array}{c}0,19 \\
\mathrm{~N}_{\mathrm{L}}^{* *} ; \mathrm{N}_{\mathrm{Q}}^{* * *} \\
\mathrm{~N}_{\mathrm{L}}^{* *}\end{array}$ \\
\hline $\begin{array}{l}\text { N retenu, } \\
(\mathrm{mg} / \mathrm{j})\end{array}$ & $\begin{array}{c}6 \\
9 \\
12 \\
\text { Moy. } \\
15 \\
18\end{array}$ & $\begin{array}{c}58,6 \\
92,1 \\
138,6 \\
96 \\
159 \\
168\end{array}$ & $\begin{array}{c}56,8 \\
110,0 \\
131,1 \\
99\end{array}$ & $\begin{array}{l}61,8 \\
120,7 \\
156,8 \\
113\end{array}$ & $\begin{array}{l}80,,^{\prime} \\
127,1 \\
155,0 \\
121\end{array}$ & $\begin{array}{r}64 \\
112 \\
145\end{array}$ & \\
\hline C.U.P. azoté & $\begin{array}{c}6 \\
9 \\
12 \\
\text { Moy. } \\
15 \\
18\end{array}$ & $\begin{array}{l}46,6 \\
46,9 \\
48,0 \\
47,2 \\
43,2 \\
39,9\end{array}$ & $\begin{array}{l}47,5 \\
51,2 \\
50,1 \\
49,6\end{array}$ & $\begin{array}{l}52,1 \\
56,2 \\
51,8 \\
53,4\end{array}$ & $\begin{array}{l}59,8 \\
60,2 \\
5,1,0 \\
58,0\end{array}$ & $\begin{array}{l}51,5 \\
53,6 \\
51,0\end{array}$ & $\begin{array}{c}2,26 \\
\mathrm{M}_{\mathrm{L}}^{* *}\end{array}$ \\
\hline
\end{tabular}

(1) Cf. légende tableaux 3 et 4 . Les calculs statistiques se rapportent uniquement au schéma factoriel.

La vitesse de croissance présente une évolution parabolique en fonction du taux de protéines, avec un maximum de $5,6 \mathrm{~g} / \mathrm{j}$ au taux de $\mathrm{I} 5 \mathrm{p}$. Ioo. Il en est de même de la consommation journalière de matière sèche, tandis que l'indice de consommation diminue régulièrement. Quant au C. U. P. azoté, son évolution est également curvilinéaire avec un maximum pour I $2 \mathrm{p}$. Ioo de protéines.

L'examen des résultats en fonction du taux de méthionine supplémentaire fait apparaître des différences (non significatives) selon le niveau azoté considéré. Ainsi, 1a vitesse de croissance, aux taux de 6 et 9 p. Ioo de protéines, augmente avec la dose d'extra-méthionine jusqu'à I, 5 p. Ioo, alors qu'au taux de I2 p. roo de matières 
azotées, elle présente un maximum pour I p. Ioo de méthionine supplémentaire. Cette vitesse de croissance maximum $(5,7 \mathrm{~g} / \mathrm{j})$ est légèrement supérieure à celle obtenue en faisant varier simplement le taux azoté $(5,6 \mathrm{~g} / \mathrm{j}$ pour I5 $\mathrm{p}$. Ioo de protéines) ; les teneurs correspondantes en acides aminés soufrés sont respectivement 0,54 et 0,52 en pourcentage de la ration. Pour provoquer cette amélioration de la croissance, la consommation journalière de matière sèche a augmenté très faiblement; il en résulte une diminution importante de l'indice de consommation, tandis que le C. U. P. azoté croît linéairement avec le taux de méthionine supplémentaire.

TABIEAU 7

Expérience B : Résultats de composition corporelle

\begin{tabular}{|c|c|c|c|c|c|c|c|}
\hline \multirow{2}{*}{ Critère } & \multirow{2}{*}{$\begin{array}{c}\text { Protéines } \\
\%\end{array}$} & \multicolumn{5}{|c|}{ DL-méthionine supplémentaire p. 100 protéines } & \multirow{2}{*}{$\begin{array}{c}\text { Signification } \\
\text { statistique } \\
s_{x}\left({ }^{1}\right)\end{array}$} \\
\hline & & 0 & 0,5 & 1 & 1,5 & moy. & \\
\hline Protéines. \% & $\begin{array}{c}6 \\
9 \\
12 \\
M o y . \\
16 \\
18\end{array}$ & $\begin{array}{l}17,6 \\
17,5 \\
18,2 \\
17,8 \\
18,2 \\
19,2\end{array}$ & $\begin{array}{l}16,9 \\
17,5 \\
18,6 \\
17,7\end{array}$ & $\begin{array}{l}16,6 \\
17,7 \\
17,8 \\
17,4\end{array}$ & $\begin{array}{l}17,1 \\
17,8 \\
17,9 \\
17,7\end{array}$ & $\begin{array}{l}17,1 \\
17,6 \\
18,1\end{array}$ & $\begin{array}{c}0,26 \\
\mathrm{~N}_{\mathrm{L}}{ }^{* *}\end{array}$ \\
\hline Lipides \% & $\begin{array}{c}6 \\
9 \\
12 \\
M o y . \\
16 \\
18\end{array}$ & $\begin{array}{l}14,3 \\
15,8 \\
14,1 \\
14,6 \\
14,4 \\
12,3\end{array}$ & $\begin{array}{l}17,7 \\
16,6 \\
13,3 \\
15,9\end{array}$ & $\begin{array}{l}18,6 \\
15,3 \\
17,0 \\
17,0\end{array}$ & $\begin{array}{l}17,0 \\
16,2 \\
14,8 \\
16,0\end{array}$ & $\begin{array}{l}16,9 \\
16,9 \\
14,8\end{array}$ & $\begin{array}{c}1,12 \\
N_{\mathrm{L}}^{*} \\
\mathrm{M}_{\mathrm{L}}(0,10) \\
\mathrm{M}_{\mathrm{Q}}(0,10)\end{array}$ \\
\hline
\end{tabular}

(1) Cf. légende tableau 3.
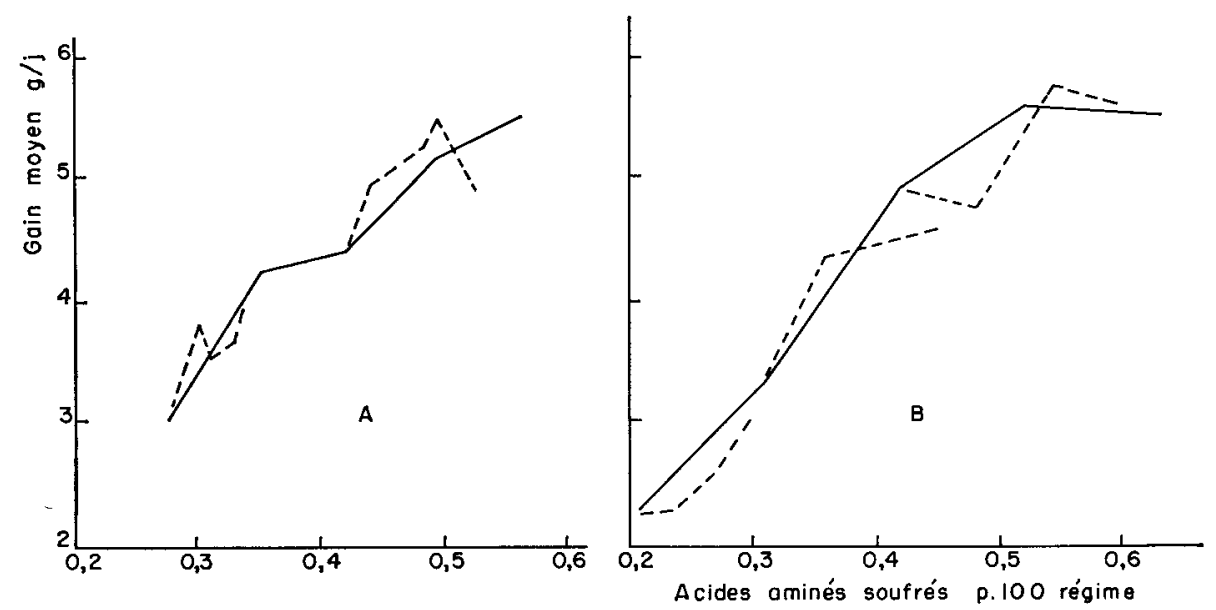

FIG. I. - Évolution du gain moyen joumalier après 28 jours en fonction du taux d'acides aminés soufrés dans le régime

A: Expérience A B: Expérience B 
En ce qui concerne la composition corporelle (tab1. 7) 1a teneur en protéines dans la carcasse augmente linéairement lorsque croît le taux azoté, tandis que la teneur en lipides diminue. L'addition de méthionine n'entraîne aucune modification du pourcentage de protéines ; par contre, l'adiposité des carcasses est maximum au taux de I p. Ioo de DL-méthionine, correspondant à la vitesse de croissance maximum.

L'évolution de la vitesse de croissance en fonction du taux d'acides aminés soufrés dans le régime, pour les deux expériences $A$ et $B$, est illustrée dans la figure I. Il est intéressant de constater que le gain de poids est sensiblement identique avec des régimes dont le taux de matières azotées est différent, mais dont le taux d'acides aminés soufrés est le même suivant qu'ils sont supplémentés ou non.

\section{DISCUSSION}

Le besoin en acides aminés soufrés chez le Rat blanc en croissance a été déterminé selon diverses modalités. Utilisant des régimes synthétiques constitués d'un mélange d'acides aminés libres, totalisant io p. Ioo du régime, RosE et ses collaborateurs (ROSE, I938; WRETLIND et Rose, I950; WoMACK et ROSE, I94I) ont montré que le taux minimum d'acides aminés soufrés, correspondant à une croissance et une rétention azotée maximum, s'élève à 0,60 p. Ioo de la ration, la cystine pouvant remplacer le sixième du total (méthionine + cystine). De leur côté, RAO et al. (I959) ont introduit une fraction des acides aminés sous forme d'une protéine naturelle $(5 \mathrm{p}$. Ioo de caséine), le complément étant apporté à l'état libre et le taux de matières azotées ajusté à Io p. Ioo ; le besoin en acides aminés soufrés ainsi déterminé représente 0,49 p. Ioo de la ration; l'apport de méthionine doit constituer au minimum o, 44 p. Ioo du régime lorsque le besoin en cystine est largement couvert (RAO et al., I96I). Opérant également en présence d'un excès de cystine de niveau non précisé, SchWEIGERT et GUTHNECK (I954) ont estimé le besoin du Rat en méthionine à $0,22 \mathrm{p}$. Ioo d'un régime à base de caséine; BARNes et al. (Ig62) 1'ont évalué à o,I7-0,I9 p. Ioo de la ration à partir de protéines isolées de soja. Enfin, Wili,iams et al. (I954), utilisant les résultats d'analyse des carcasses, ont chiffré le besoin en acides aminés soufrés du Rat à 0,43 p. Ioo de la ration.

Au cours de la présente étude a été utilisée comme source azotée unique la farine de poisson de Norvège. L e calcul du pourcentage de déficit des différents acides aminés indispensables par rapport au besoin du Rat ou par rapport à une protéine de référence, selon la méthode de MITCHELL et BI,Ock (I946), fait apparaître un déficit primaire en acides aminés soufrés. Les résultats obtenus confirment l'influence bénéfique d'une supplémentation de la farine de poisson par la méthionine qui a d'ailleurs été mise en évidence par d'autres auteurs (NJAA, I96I ; ChALUPA et FISH, I963). La meilleure croissance, qu'on peut considérer comme maximum, est obtenue en ajoutant dans les protéines du régime à $\mathrm{I} 2 \mathrm{p}$. Ioo de matières azotées, soit o,6 p. Ioo (expérience A), soit I,0 p. Ioo (expérience B) de DL-méthionine. Les valeurs correspondantes pour le besoin en acides aminés soufrés à partir de la farine de poisson, exprimées en pourcentage du régime, présentent entre elles un écart d'environ Io p. Ioo (0,49 p. Ioo dans l'expérience A; 0,54 p. Ioo dans l'expérience B); l'expérience A confirme les résultats de Rao et al. (I959, I96I). Par contre, Rose 
et al. (Womack et Rose, I94I ; Wrethind et Rose, I950) ont obtenu une valeur nettement plus élevée $(0,60 \mathrm{p}$. I0o), bien que la croissance obtenue ait été plus faible que dans nos essais ou ceux de RAO et al. (I96I) : on peut supposer que les régimes synthétiques utilisés par ROSE et ses collaborateurs, mis au point beaucoup plus anciennement, présentaient d'autres facteurs limitants, soit azotés, soit vitaminiques, soit minéraux.

Quoi qu'il en soit, le mode d'expression du besoin en pourcentage du régime n'est correct que si l'on fait l'hypothèse que le niveau de consommation est invariable à un poids donné d'un individu à l'autre et d'un régime à un autre. Or, on sait qu'il n'en est rien et que notamment la composition des régimes intervient dans une large mesure sur le niveau de consommation : celui-ci est en relation inverse avec la densité énergétique du régime chez le Rat (HENRY et RÉRAT, I965); il peut également varier avec la nature des sucres du régime (HARPER et ElvEHJEM, I957; HARPER et SPIVEy, I958). Il semble logique par conséquent d'exprimer le besoin d'acides aminés en quantités nécessaires par jour et non plus en pourcentage du régime. Dans ces conditions, l'apport journalier nécessaire d'acides aminés soufrés passe de $70 \mathrm{mg}$ dans l'expérience $\mathrm{A}$ à $75 \mathrm{mg}$ dans l'expérience $\mathrm{B}$ ou à $63 \mathrm{mg}$ dans l'expérience de RAO et al. (I959), la différence entre ces valeurs pouvant être expliquée par l'origine génétique des animaux utilisés dont la vitesse de croissance est plus ou moins rapide.

Dans la détermination du besoin en acides aminés soufrés, il n'a pas été tenu compte séparément de la méthionine et de la cystine. La raison en est que dans les protéines de poisson, la cystine représente $25 \mathrm{p}$. Ioo du total, de sorte que l'addition de méthionine permet đ’obtenir le besoin global en acides aminés soufrés, mais non pas le besoin minimum en méthionine seule. A ce sujet, il faut rappeler que contrairement aux premiers résultats de Rose et de son équipe (Womack et RosE, I94I; WRETLIND et ROSE, I950), l'apport de cystine peut représenter d'après RAO et al. (I96r) près de $70 \mathrm{p}$. Ioo de l'apport des amino acides soufrés. Le taux de cystine des régimes que nous avons utilisés n'est donc pas excessif.

L'évolution de la vitesse de croissance en fonction du taux de protéines et du taux de DL-méthionine supplémentaire a fait apparaître une diminution de la quantité totale d'acides aminés soufrés nécessaires pour l'équilibre des protéines de poisson lorsque le taux de ces dernières augmente : de plus de 5 p. Ioo par rapport aux protéines pour $6 \mathrm{p}$. Ioo de protéines à $4-4,5 \mathrm{p}$. IOO pour I $2 \mathrm{p}$. IOO et à $3,5 \mathrm{p}$. IOO pour $15 \mathrm{p}$. roo. Il s'agit là d'un phénomène général qui se retrouve pour d'autres acides aminés, comme la lysine (RÉRAT et HENRY, I964) et chez d'autres espèces (Almouist, I959). Il est bien évident que, lorsqu'on augmente le taux azoté, les déficits en différents acides aminés par rapport aux besoins (tabl. 8) ont tendance à disparaitre les uns après les autres et seul subsiste le déficit du facteur limitant primaire; si, à partir de ce taux critique de matières azotées, on augmente encore la teneur en azote du régime, alors le déficit du facteur limitant primaire se résorbe et la dose additionnelle d'acide aminé synthétique permettant de combler ce déficit diminue pour s'annuler à un taux donné de matières azotées. Dans le cas présent, étant donné que la réponse de croissance obtenue en supplémentant le régime à I 2 p. roo de protéines est supérieure à celle provoquée par les régimes à I 5 ou I 8 p. Ioo de protéines, on peut en déduire que l'apport de facteur limitant secondaire par le régime à $\mathrm{I} 2 \mathrm{p}$. Ioo de protéines de poisson est largement suffisant. Il est difficile 
de préciser quel est le facteur limitant secondaire : si l'on tient compte des résultats de RAo et al. (I959), il peut s'agir soit de la lysine, soit de la thréonine, soit de 1'isoleucine, soit plus probablement de ces trois acides aminés ensemble.

L'évolution du besoin en acides aminés soufrés avec l'âge ne peut être étudiée de façon précise dans ces expériences du fait d'une part de l'espacement trop grand des taux azotés et, d'autre part, de l'influence que peut avoir le traitement subi pendant une première période expérimentale sur les résultats obtenus au cours de la deuxième période expérimentale. Notons simplement que, dans l'expérience $A$,

TABLEAU 8

Composition (en p. 100) en amino acides des régimes aux différents taux azotés et déficits par rapport aux besoins

(RAO et al., 1959)

\begin{tabular}{|c|c|c|c|c|}
\hline \multirow{2}{*}{$\begin{array}{l}\text { Source azotée du régime } \\
\text { Taux de matières azotées }\end{array}$} & \multicolumn{3}{|c|}{ Farine de poisson } & \multirow{2}{*}{$\begin{array}{r}\begin{array}{c}\text { Caséine }+ \\
\text { amino acides }\end{array} \\
10\end{array}$} \\
\hline & 6 & 9 & 12 & \\
\hline Lysine $\ldots \ldots \ldots \ldots \ldots$. . . & $0,46(49)^{*}$ & $0,69(23)^{*}$ & 0,92 & 0,9 \\
\hline Histidine $\ldots \ldots \ldots \ldots$ & $0,14(33)^{*}$ & 0,21 & 0,28 & 0,21 \\
\hline Tryptophane .......... & - & - & - & 0,11 \\
\hline Isoleucine $\ldots \ldots \ldots \ldots$ & $0,31(44)^{*}$ & $0,46(16)^{*}$ & 0,62 & 0,55 \\
\hline Leucine ............. & $0,48(30)^{*}$ & 0,72 & 0,96 & 0,69 \\
\hline Valine $\ldots \ldots \ldots \ldots \ldots$ & $0,37(34)^{*}$ & 0,55 & 0,74 & 0,56 \\
\hline Thréonine ........... & $0,28(45)^{*}$ & $0,42 \quad(18)^{*}$ & 0,56 & 0,51 \\
\hline Méthionine + cystine ... & $0,21 \quad(57)^{*}$ & $0,32(35)^{*}$ & $0,42(14)^{*}$ & 0,49 \\
\hline Phénylalanine + tyrosine & $0,47(35)^{*}$ & 0,70 & 0,94 & 0,72 \\
\hline
\end{tabular}

$\left(^{*}\right)$ Les chiffres entre parenthèses indiquent le pourcentage de déficit par rapport aux besoins.

lorsque les animaux prennent de l'âge, le pourcentage nécessaire d'acides aminés soufrés dans le régime diminue, mais de façon moins nette que ne l'ont observé Hartsook et Mrtchel L (I956); il passe de 0,49 p. Ioo (lot A o,6) pendant les quatre premières semaines d'expérience à $0,47 \mathrm{p}$. Ioo (lot $\mathrm{A} 0,4)$ pendant les quatre dernières semaines ; en outre, contrairement à ce qui a été trouvé par ces auteurs, le pourcentage nécessaire d'acides aminés soufrés dans les protéines n'augmente pas avec l'âge ; enfin, la quantité journalière nécessaire passe de $70 \mathrm{mg}$ pour un gain de poids de $5,44 \mathrm{~g} / \mathrm{j}$ pendant la première période à $82 \mathrm{mg}$ pour un gain de poids de $4 \mathrm{~g} / \mathrm{j}$ (lot $\mathrm{A} 0,4)$ pendant la deuxième période, les valeurs correspondantes de HarTsook et Mitchel. (I956) étant respectivement $72 \mathrm{mg}$ et $54 \mathrm{mg}$. Ici encore, il faut attribuer en partie cette différence de comportement à l'origine génétique, les animaux utilisés par les auteurs américains semblant plus précoces (gain de $7,4 \mathrm{~g}$ par jour à 40 jours) et d'un poids adulte plus faible que les nôtres; en outre, la synthèse de kératine, dont dépend le niveau du besoin de cystine, n'est pas nécessairement la même entre les différentes souches de rats.

En définitive, lorsqu'on supplémente la farine de poisson par la DL-méthionine, à différents taux dans le régime, la vitesse de croissance maximum est atteinte avec 
I2 p. Ioo de protéines et 0,6 à I p. Ioo de DL-méthionine supplémentaire par rapport aux protéines, alors qu'en régime non supplémenté il faut I5 à I 6 p. Ioo de matières azotées pour obtenir la même vitesse de croissance. On peut en déduire que le besoin du Rat en acides aminés soufrés, dans les conditions de croissance maximum, représente encore 0,49 et $0,54 \mathrm{p}$. Ioo d'un régime renfermant 4 I5o Kcal digestibles $/ \mathrm{kg}$, soit en moyenne 70 à $75 \mathrm{mg}$ par jour dans l'intervalle de poids $50 \mathrm{~g}-300 \mathrm{~g}$.

Reçu pour publication en juillet 1965 .

\section{SUMMARY}

ESTIMATION OF TIE SULPHUR AMINO ACID REQUIREMENT

OF THE GROWING WIITE RAT

Sulphur amino acid requirement of growing white rats was estimated in 2 experiments based on the principle of supplementing a natural protein, Norwegian herring meal, with methionine, the first limiting amino acid of that protein. In the first experiment (A), lasting 56 days, male Wistar rats of $7 \mathrm{I} g$ initial weight were given diets with 8, I0, I2, I4 or 16 per cent protein from fish. With 8 and i 2 per cent increasing amounts of synthetic DL-methionine were added in relation to the protein content $(0,0.2,0.4,0.6$ and 0.8 per cent of the protein). The second experiment $(\mathrm{B})$ lasting 28 days, was of similar design with rats of $59 \mathrm{~g}$ at the start. Four levels of supplementary DL-methionine (o, $0.5,1.0$ and 1.5 per cent of protein) were given with diets with 6,9 or 12 per cent fish protein. As in the first experiment, diets with $\mathrm{I}_{5}$ and $\mathrm{i} 8$ per cent protein also were given to obtain maximum performance by changes only in protein content. The criteria chosen were rate of growth and retention of nitrogen estimated from analysis of carcases.

When methionine was added to diets with from 6 to 12 per cent crude protein growth rate and retention of nitrogen were significantly improved. Maximum growth after 28 days, $5.4 \mathrm{~g}$ per day in the first experiment and $5.7 \mathrm{~g}$ in the second, were on the diet with 12 per cent protein, and 0.6 and 1.0 per cent extra DL-methionine in the respective experiments. That growth rate was practically the same as when only protein content was changed, $5.5 \mathrm{~g}$ per day with 16 per cent crude protein in the first experiment and $5.6 \mathrm{~g}$ with $\mathrm{I} 5$ per cent in the second. The amount of sulphur amino acids (methionine + cystine) needed to balance the diet decreased, relative to the protein, as the protein content increased, 5 and 4 to 4.5 per cent, respectively, for diets with 6 and 12 per cent protein. The requirement for maximum growth was between 0.49 and 0.54 per cent in a diet supplying $4 \mathrm{I} 50 \mathrm{kcal}$ digestible energy per $\mathrm{kg}$, or on average 70 to $75 \mathrm{mg}$ per day in the interval from 50 to $300 \mathrm{~g}$.

\section{RÉFÉRENCES BIBLIOGRAPHIQUES}

Barnej R. H., Finla G., Kwong E., igs2. Methionina supplementation of processed Soybeans in the rat. J. Nutr. $77,278-284$.

Bressani R., Mertz I.. T., i958. Relationship of protein level to the minimum lysine requirement of the rat. J. Nutr. 65, $48 \mathrm{I}-49 \mathrm{I}$.

Chalupa W., Fish H., ig63. Comparative protein evaluation studies by carcass retention and nitrogen balance methods. J. Nutr., 81, 1 39-146.

Fauconneau G., 1964. Méthodes modernes de dosage des acides aminés des tourteaux. Revue Francaise des Corps gras (numéro spécial), I48-I6I.

Geiger E., Borgstrom G., I962. Fish protein - Nutritive aspects. In Borgstrom G., Fish as Food, vol. 11, Nutrition, sanitation and utilization, 29-1 I4, Acad. Press, New York.

Harper A. E., Elvehjem C. A., I957. A review of the effects of different carbohydrates on vitamin and amino acid requirements. J. Agric. food Chem., 5, $754^{-7}-58$.

Harper A. E., Spivey H. E., i 958 . Relationship between food intake and osmotic effect of dietary carbohydrate. Amer. J. Physiol., 193, 483-487.

Hartsook E. W., Mitciell H. H., 1956. The effect of age on the protein and methionine requirement of the rat. $J$. Nutr., 60, 173-I95. 
Henry Y., Rérat A., Ig63. Étude de l'ingestion spontanée d'éléments énergétiques et de protéines chez le Rat en croissance par la méthode du libre choix. Ann. Biol. Anim. Bioch. Biophys., 3, io3-I 7 .

HENRY Y., RÉrit A., 1965. Évolution de l'ingestion spontanée de principes énergétiques en fonction de la vitesse de croissance et de la protéinogenèse chez le Rat blanc. Amino Acides - Peptides - Protéines, cahier n ${ }^{\circ} 6, A, E . C$., Commentry.

Mertz E. T., Beeson W. M., Jackson H. D., i952. Classification of essential amino acids for the weanling pig. Arch. Biochem. Biophys. 38, I $2 \mathrm{I}-\mathrm{I} 28$.

Mitcielel H. II., Block R. J., I946. Some relationships between the amino acid contents of proteins and their nutritive values for the rat. J. Biol. Chem. 163, 599-620.

NJAA L. R., I96r. Effect of supplementing with methionine, cystine and derivatives of thiazolidine - 4carboxylic acid on the nutritive value of herring-meal protein. J. Sci. Agric. 12, 757-763.

Piox R., De Belsunce C., ig63. Influence de la conduite de séchage à la flamme sur l'efficacité des protéines de farines de Hareng. Composition en acides aminés. Inn. Biol. anim. Bioch. Biophys., 3 (No hors série, I), 65.

Rama RaO P. 33., Chatam Metta V., Connor Jonnson B., I959. The amino acid composition and the nutritive value of proteins. I. Essential amino acid requirement of the growing rat. J. Nutr., 69, 387391.

Rama Rao P. B., Norton H. W., Convor Johnson B., igsr. The amino acid composition and nutritive value of protein. IV. Phenylakinine, tyrosine, methionine and cystine requirement of the growing rat. J. Nutr. $73,38-42$.

Rérat A., Lougnon J., 1963. Valeur nutritionnelle des protéines. IV. Influence de la conduite de séchage à la flamme sur l'efficacté des protéines de farines de hareng. Études sur le Rat. Ann. Biol. anim. Bioch. Biophys., 3, (ñ ${ }^{\circ}$ hors série), $7 \mathrm{I}-74$.

Rérat A., HEnry Y., ig63. Besoin en lysine du Rat en croissance : principe d'une méthodologie et résultats expérimentaux. C. R. Acad. Sci., Paris, 257, 3 0.45-3 048.

Rose W. C., I938. The nutritive significance of the amino acids and certain related compounds. Science 86, 298-300.

Schweigert B. S., Guthineck B. T., I954. Utilization of amino acids from foods by the rat. III. Méthionine. $J$. Nutr., 54, 333-3+3.

Terroine E. F., Valla S., I933. Valeur comparée des différents aliments protéiques dans la croissance Bull Soc. Sci. dilyg. aliment. 21, I05-1 $7+$.

Willams H. I1., Curtix L. V., Abraitaur J., Loosli J. K., Maysard L. A., i954. Estimation of growth requirement for amino acids by assay of the carcass. J. Biol. Chem., 208, 277-286.

Womack M., Rose W. C., I94I. The partial replacement of dietary methionine by cystine for purposes of growth. J. Biol. Chem., 141, 375-379.

Wretlind K. A., Rose W. C., I950. Methionine requirement for growth and utilization of its optical isomers. J. Biol. Chem., 187, 697 . 